Ann. A bille, $\operatorname{Ig}_{4} 7(3), \mathbf{r g r}-200$.

\title{
RECHERCHES SUR CERTAINES PROPRIÉTÉS BIOLOGIQUES DE LA PROPOLIS
}

\author{
Adelina DERINICI, A. POPES( \\ Laboratoire central de Controle des Produits alimentaires, Bucurst (Rommanie)
}

SOMMAIRE

Les trois échantillons de propolis testés présentent une action inhibitrice envers l'Entérocoque et divers autres microbes. Il n'y a pas d'action sur le Staphylocoque Oxford. Les résultats sont obtenus apres maintien a $37^{\circ}$ des tubes de bouillon + propolis ensememés arec différents microbes et passés ensuite sur gélose : les auteurs ont constaté également une action inhibitrice à la dilution de $\mathrm{r} /$ ro sur la germination des semences de chanve. Dautre part. on constate une réduction de l'aérofore interne du rucher par rapport à celle du verger et de la ville.

\section{INTRODUC'TION}

La propolis peut être considérée comme un moyen naturel de défense des abeilles. C'est un produit qui contient de la cire, du pollen et des substances résineuses qui sont récoltées sur les bourgeons ou sur l'écorce de certains arbres, soumises ensuite à l'action de certaines sécrétions glandulaires et enfin régurgitées sous forme d'une pâte consistante. La composition de la propolis p. Ioo est la suivante :

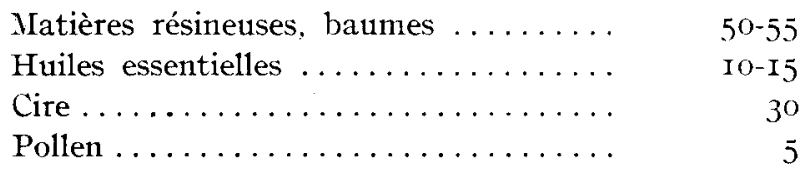

La propolis sert aux abeilles comme matériau de construction pour la réduction du trou de vol, pour combler les fentes des ruches, pour fixer les cadres. Elle sert également à enduire les alvéoles dans lesquels doivent être déposés les cufs.

Les huiles essentielles et les baumes qui entrent dans la composition de la propolis lui confèrent des propriétés antibactériennes et antifongiques. Ces propriétés se manifestent même lorsque la propolis est étalée en mince couche isolante, sur 
certains intrus, souris ou grands papillons, tués par les abeilles mais dont les ca davres: ne peuvent être évacués de la ruche.

Les propriétés antiseptiques de la propolis ont été confirmées par des recherches cliniques et de laboratoire.

Parmi les travaux qui ont été publiés, les uns concernent les propriétés antibactériennes (Iolrich N. P., Derevici A., Petrescu A., I964; Karimova Z. H., i960; Keller F. E., Prlonnicenko E. C., ig60 ; Krvaikina V. P., ig60 ; Lavie P., ig60; Lavie P., ig6o; Marta M., Mateesco T., Baiaci T., Popa A., I959; Salmakov M. K., I960; Rabriovici I. M., I960; VhRGE J., Ig6I), d'autres les propriétés antifongiques (LAviE P., Ig60) ou virulicides (IOIRICH N. P., I960; SEIAARSKI V. A., I960) de la propolis. LAVIE P. (I960) a consacré à ce produit des recherches approfondies et a proposé la détermination de l'action antibiotique par mesure de l'inhibition constatée sur une culture de Bacillus subtilis.

Gonver et LAvie (I960) étudiant 1'action inhibitrice des extraits de divers produits de la ruche (cire, pollen, gelée royale, corps total des abeilles, propolis) sur la germination du riz et des tubercules de pommes de terre, concluent que la propolis est la plus active.

La connaissance des propriétés biologiques de la propolis a permis, au cours de ces dernières années, son utilisation avec des résultats satisfaisants en thérapeutique humaine et vétérinaire, en dermatologie, gynécologie, stomatologie et dans le traitement de diverses maladies infectieuses.

Karimova (ig60), Stepanova (ig60), Brusilovski (ig6o), Marcenko (ig6o), Muhamediarof (ig60), Kazakov et coll. (I960), Salmakov (I960), Szelarki (Ig60), Idrisova (I960), Cianesev (I960), Giagoleva (I960), KuleEV (I960), Kirkunov (I96r), se sont occupés de ces problèmes.

L'intérêt de ces observations sur la propolis nous a incités à entreprendre des recherches sur les propriétés biologiques de ce produit autochtone, encore peu étudié.

Nous nous sommes proposés d'étudier quelques échantillons de propolis provenant de notre pays, comparativement avec une propolis qui nous fut envoyée d'U. R. S. S. par le Pr IOIRICH.

Nous avons étudié l'action inhibitrice sur le développement de certains microbes, sur la germination de certaines semences et également l'action sur l'aéroflore d'un rucher placé en_plein air.

\section{MATÉRIEL, ET TE.CHNIQUE}

La propolis native, ayant la consistance du mastic lorsqu'elle est sèche, est coupée ou effritée en tout petits morceaux, dont on introduit $250 \mathrm{~g}$ dans un flacon contenant $\mathrm{I}$ litre d'alcool éthylique à $96^{\circ}$, et qu'on ferme hermétiquement. Le flacon est maintenu dans l'obscurité et agité journellement pendant 3 à 5 jours. L'alcool prenant une teinte brun-foncé est utilisé après filtration, les substances insolubles dans l'alcool se déposent. On prépare des extraits alcooliques avec deux échantillons de propolis roumaine notés $R_{1}$ et $R_{2}$ et un échantillon de propolis soviétique noté "U.R.S.S. " La quantité de substance sèche contenue dans les différénts échantillons de propolis est consignée dans le tableau $\mathrm{I}$ exprimant le pourcentage pour $100 \mathrm{~g}$ et $100 \mathrm{ml}$. On constate un pourcentage similaire pour les différents échantillons utilisés.

Pour l'interprétation des résultats, nous avons étudié - afin d'éviter d'éventuelles erreurs l'influence de l'alcool sur la culture des microbes destinés à servir dans nos expériences.

L'extrait alcoolique de propolis est ajouté au boullon de culture à la concentration de $\mathrm{I} / \mathrm{ro}$. 
Le témoin alcool-bouillon est prèparé à la même concentration. Il a été établi que lalcool, à cette concentration, n'empêche pas la culture de certains microbes $\left(^{(1)}\right.$, tels que :
Staphylocoque Oxford
Escherichia coli type $\mathrm{O}_{26}$
$B$. subtilis mesentericus
S. enteritidis GÄRTNER 5168 .

Un autre témoin (extrait alcoolique ensemencé sur bouillon et passage sur gélose inclinée) établit la stérilité de l'extrait de propolis.

TABI,EAU I

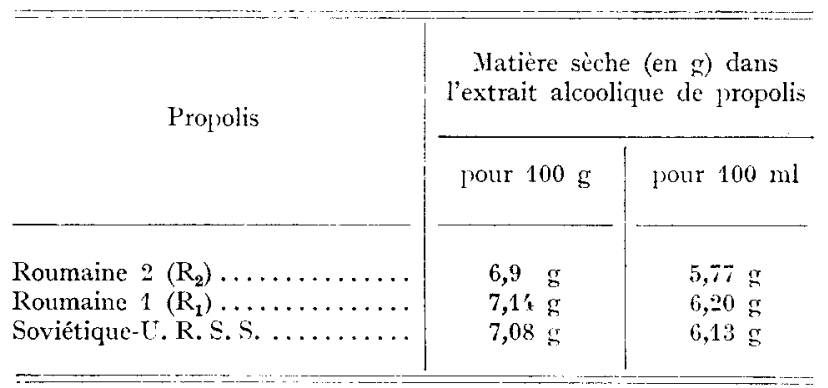

Dans le but d'obtenir la concentration optima de l'extrait alcoolique, on ajoute différentes quantités d'extrait au bouillon dans lequel doivent être ensemencés divers microbes. On introduit dans le tube de bouillon les quantités de cet extrait permettant d'obtenir une concentration finale de $\mathrm{I} / \mathrm{IO}, \mathrm{I} / 20, \mathrm{I} / 4 \mathrm{O}, \mathrm{I} / 80$. Après maintien des tubes de bouillon pendant 48 heures à $37^{\circ} \mathrm{C}$, on effectue des passages sur gélose inclinée. La culture en bouillon ne permet pas, en effet, la lecture à cause de l'aspect lactescent opaque, déterminé par l'addition de propolis.

\section{PRINCIPAUX RÉSULTATS}

\section{I. - Action antibactérienne de la propolis}

Des expériences préliminaires avec l'extrait alcoolique au I/Io ajouté aux cultures de Staphylocoque Oxford, B. subtilis mesentericus, S. enteritidis GÄRTNER 5 I 68 et Escherichia coli, démontrent une action inhibitrice mais uniquement envers les deux derniers microbes. Les expériences faites avec une autre série de microbes en utilisant la propolis "U. R. S. S. " aux concentrations de I/Io et I/20 ont montré, par ailleurs, une inhibition des microbes suivants : S. enteritidis GÄRTNER $5 \mathbf{I} 68$. E. coli type 026 et oII, Sh. dysenteriae 2 HELLY, S. cholerae suis I79, phase I, S. typhi TO gor, ainsi que de l'entérocoque (Str. faecalis) mais pas d'inhibition du Staphylocoque Oxford ni de E. subtilis mesentericus (tabl. 2).

I'influence de la température et du temps de contact est étudiée dans une autre série d'expériences en utilisant l'extrait alcoolique de la propolis $\mathrm{R}_{1}$. Les passages sur gélose inclinée sont effectués à divers intervalles de temps à partir des suspensions de microbes en culture sur bouillon + propolis $1 /$ Io, maintenues à la température du laboratoire $\left(22^{\circ}\right)$ ou à l'étuve.

Durant la première heure, on fait des prélèvements de Io en Io minutes; les

(1) Ces microbes proviennent de la collection de l'Institut Dr J.-C. Cantacuzino. 
24 heures suivantes, d'heure en heure. D'autres tubes sont ensentencés sur gélose avec la même série de suspensions de microbes en culture sur bouillon - propolis maintenues pendant 24 heures à l'étude à $37^{\circ}$. I,es tubes de gélose sont ensuite conservés $4^{8}$ heures à l'étuve.

\section{TABI,EAU 2}

Letion de lestrait alcoolique de propolis "U.R.S.S." in bouillon $1 / 10$ et 1/20, at passage sur gilose, sur la cullure de cerains microbes. Incubation it lítue'e

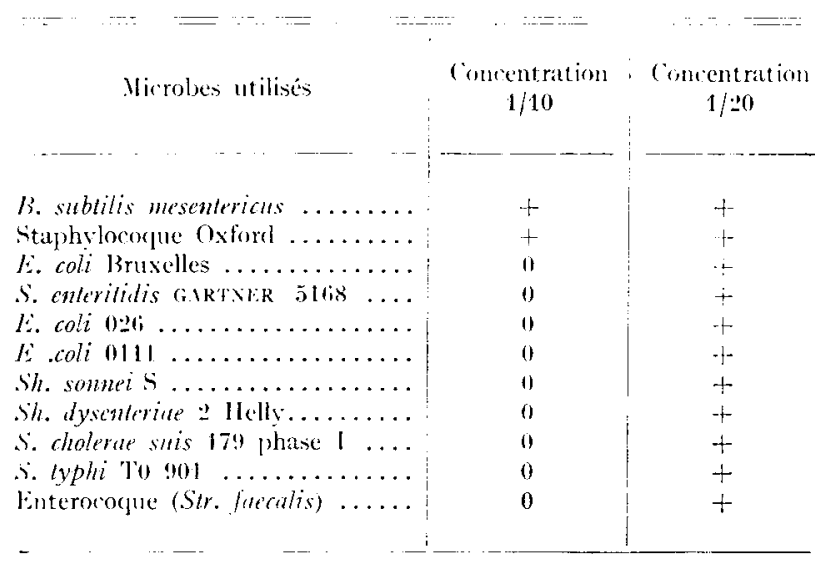

0 :-: cultures négatives: :

$+=$ cultures positives apres a hemes à 370.

\section{TABI,EAU 3}

Ation de la propolis $R_{1}$ sur certains microbes

dans diffirentes conditions de tempirature at durée de contact

$d u$ milange microbes + bouillon + propolis $1 / 10$, aint le passage sur gilose

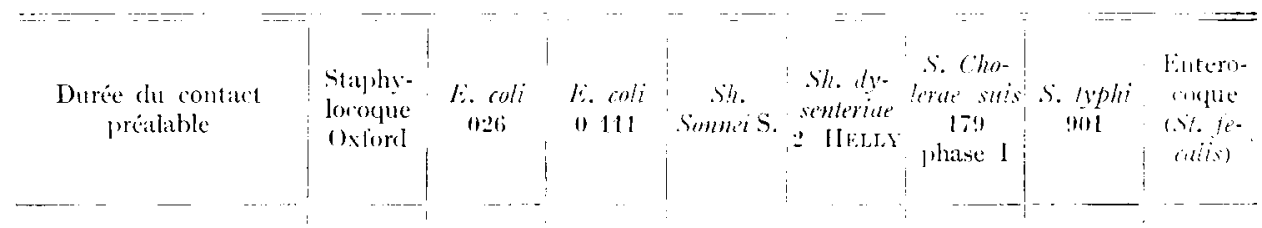

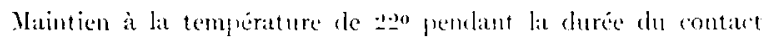

$10 \mathrm{mn}-[\mathrm{xil} \mathrm{mn} \ldots \ldots \ldots$.

it h-15 h

$16 \mathrm{~h}-9 \mathrm{~g}$ h

$\pm \mathrm{h}$

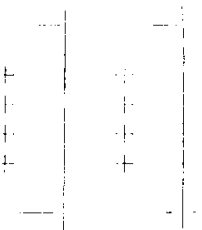

Maintien à la température de $: 37^{\circ}$ pendant la dure du contad

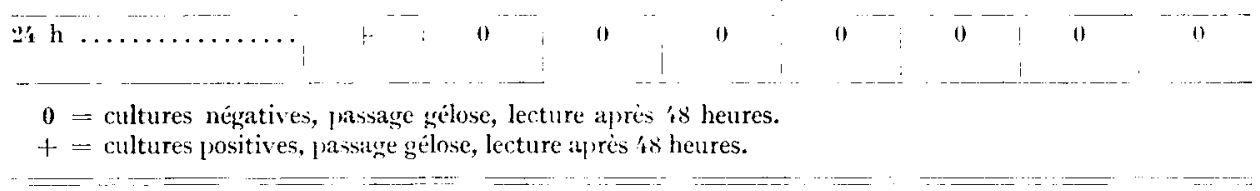


Du tableau 3, il ressort une action inhibitrice sur la Sh. dysenteriae 2 HELI.Y et sur l'entérocoque (Str. faecalis), à partir de 4 heures de contact avec la série ensemencée au moyen de la culture (bouillon + propolis I/IO) maintenue à la température du laboratoire. Pas d'action inhibitrice pour le staphylocoque Oxford $E$. coli 026 et ori I Sh. sonnei S, E. cholerae suis, S. typhi TO gor.

Le maintien du bouillon ensemencé + propolis à $37^{\circ}$ pendant 24 à 25 heures, détermine l'inhibition des microbes cités à l'exception du staphylocoque Oxford.

Ces résultats nous ont incité à rechercher si ces différences de comportement ne pourraient pas être attribuées à certaines qualités de la propolis. Mentionnons que la propolis $R_{1}$ utilisée dans l'expérience antérieure avait été malaxée et chauffée à $60^{\circ}$ pendant 15 minutes. Son aspect était très homogène et sa couleur noire, contrairement aux deux autres séries de propolis, non homogènes et de couleur brune.

L'action inhibitrice de la propolis a été étudiée en fonction de la qualité de l'échantillon, de la température et de la durée de contact.

Trois échantillons de propolis "U.R.S.S. ", $R_{1}$ et $R_{2}$ ont été expérimentés sur E. Coli or II, S. cholerae suis I79, phase I et entérocoque (Str. faecalis).

E,n conclusion de ces essais, on peut dire que ces trois échantillons présentent une action inhibitrice envers l'Entérocoque, que le mélange microbes-bouillon-propolis soit maintenu à la température du laboratoire (I I, 24 ou 30 heures) ou bien 24 heures à l'étuve à $37^{\circ}$.

On ne constate d'inhibition après II heures et 24 heures de contact pour $E$. Coli ori l et $S$. cholerae suis I79, phase I, avec aucun des trois échantillons (à $22^{\circ}$ ); les propolis "U. R.S.S. " et $\mathrm{R}_{2}$ exercent une inhibition dans tous les tubes ensemencés, après 30 heures de contact à la température du laboratoire (tabl, 4)

\section{2. - Action de la propolis sur la germination de certaines semences}

Nous avons mentionné plus haut les travaux qui mettent en évidence l'action antigerminative de la propolis, propriété attribuée à la présence d'un facteur antibiotique dans la composition (LAvie P., ig60; Marcenko A. I., I960).

I)ans le but de confirmer cette propriété chez les échantillons de propolis utilisés par nous, nous avons entrepris des expériences comparatives avec la propolis roumaine $R_{2}$ et soviétique "U. R. S. S. ".

Le choix des semences à utiliser a été fait après avoir établi la durée de la germination de séries de 50 graines soumises à la germination dans un double volume d'eau en maintenant les flacons dans les mêmes conditions de température, humidité et obscurité, à 1'étuve à $37^{\circ}$. Les expériences préliminaires avec les semences suivantes: Avoine (Avena sativa), Orge (Hordeum vulgare), Blé (Triticum dicoccum), Volubilis (Ipomoea purpura), et Chanvre (Canabis sativa) ont déterminé le choix du chanvre dont la durée de germination s'est avérée plus courte. Ia germination est notée définitivement $1 \mathrm{e} 3^{\mathrm{e}}$ jour.

On a effectué quatre séries d'expériences avec la propolis soviétique et roumaine $\mathrm{R}_{2}$, introduisant l'extrait alcoolique de manière à obtenir une dilution finale de I/IO, I/2O, I/40; afin d'exclure l'action nocive de l'alcool correspondant aux mêmes dilutions, nous avons préparé une série de témoins à l'alcool et enfin une série de témoins avec de l'eau, Les chiffres arabes du tableau 5 représentent la moyenne finale des semences germées au cours des quatre expériences.

Annales de l'Abeille. - 1964 . 


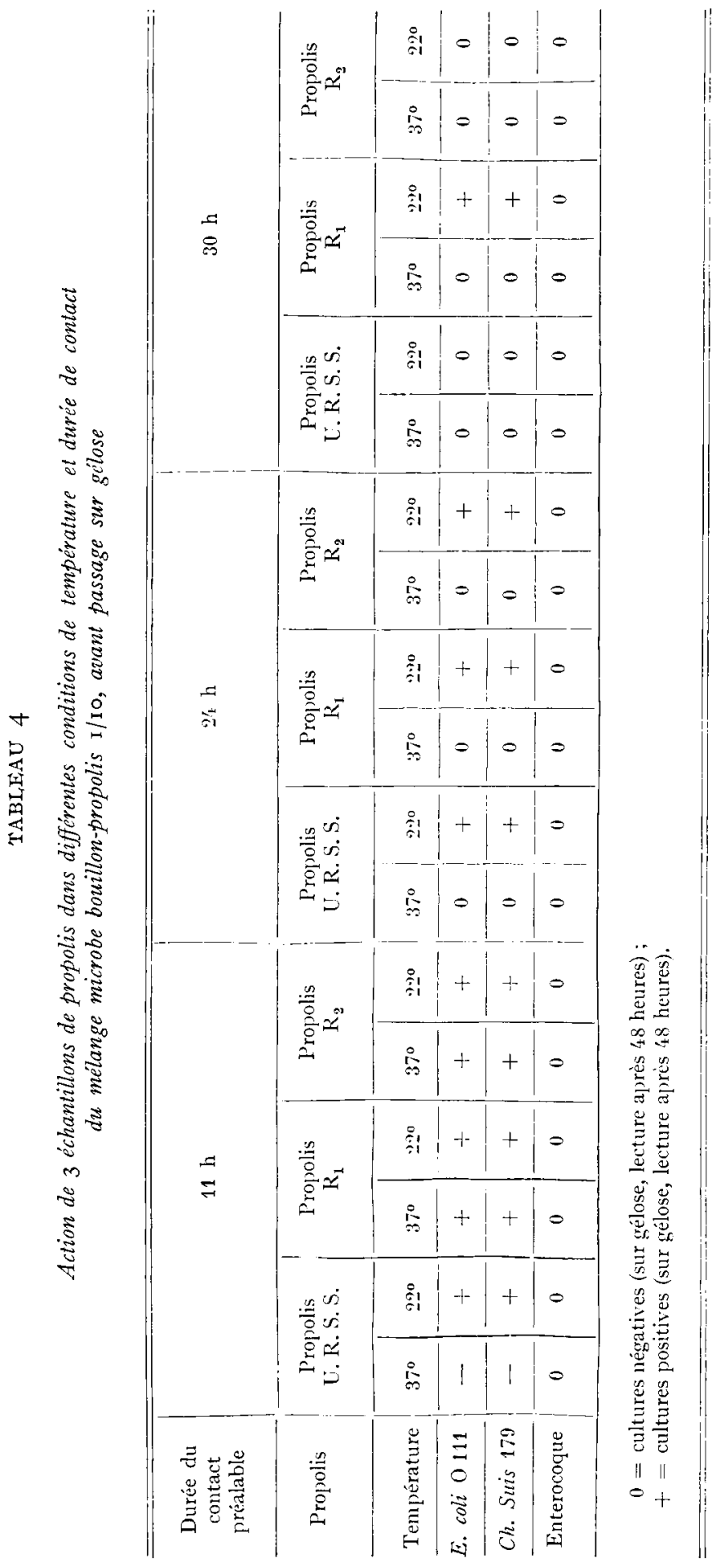


L'action inhibitrice de la propolis sur la germination - proportionnelle à la concentration - a été démontrée mais uniquement pour les dilutions I/Io et $\mathrm{r} / 20$. $L_{1}$ a propolis roumaine $R_{2}$, à la concentration de $\mathrm{r} / \mathrm{IO}$, se montre plus active que la propolis soviétique; absence d'inhibition pour la dilution $\mathrm{I} / 4 \mathrm{O}$ et la solution témoin eau-alcool r/Io dont les chiffres se rapprochent de cetux de l'eau (tabl. 5).

TABLEAU 5

Action de la propolis U.R.S.S. el $R_{2}$ sur la germination de certaines semences

\begin{tabular}{|c|c|c|c|c|c|c|c|c|c|c|}
\hline Dilutions & \multicolumn{4}{|c|}{ Propolis U.R.S.S. } & \multicolumn{4}{|c|}{ Propolis $R_{2}$} & \multicolumn{2}{|c|}{$\begin{array}{l}\text { Moyenne } \\
\text { semences } \\
\text { geminées }\end{array}$} \\
\hline Numéro de l'expérience & $\mathrm{I}$ & VI & VII & II & III & IV & $\mathrm{V}$ & VIII & URSS & $\mathrm{R}_{2}$ \\
\hline$\ldots \ldots$ & - & 13 & 10 & 8 & 2 & 3 & 8 & 8 & 10 & 5 \\
\hline $1 / 20 \ldots \ldots \ldots \ldots \ldots$ & 10 & 10 & 10 & 12 & 7 & 18 & 8 & 12 & 12 & 11 \\
\hline $1 / 40 \ldots \ldots \ldots \ldots \ldots$ & - & 25 & 28 & 30 & 30 & 30 & 33 & 19 & 97 & 28 \\
\hline $\begin{array}{l}\text { Témoin eau }+ \text { alcool } \\
1 / 10 \ldots \ldots \ldots \ldots \ldots\end{array}$ & 30 & 28 & 29 & 33 & 33 & 22 & 33 & 32 & 30 & 30 \\
\hline Témoin eau pure .... & 30 & 29 & 29 & 30 & 32 & 28 & 33 & 30 & 29,5 & 30 \\
\hline
\end{tabular}

Les chiffres latins représentent le numéro donné à l'expérience.

I.es chiffres arabes représentent le nombre de semences de chanvre germinées, après 3 jours de contact avec l'eau + propolis, à différentes concentrations.

\section{3. - Action de la propolis native sur l'aéroflore du rucher}

La troisième catégorie de nos expériences concerne l'influence exercée éventuellement par les substances volatiles contenues dans la propolis sur l'aéroflore présente dans l'atmosphère interne du rucher d'une ferme située en dehors de la ville de Bucarest et d'où provient la propolis $R_{2}$.

Dans ces expériences, nous avons appliqué la technique classique de sédimentation de Koch, en laissant les boîtes de Petri contenant soit le milieu à la gélose (pour les microbes), soit le milieu Ceapek (pour les moisissures) découvertes pendant ro minutes aux endroits choisis pour les expériences. En vue d'obtenir une moyenne plus précise, on a fait des expériences doubles, avec prélèvements le matin et l'aprèsmidi.

Des prélèvements étaient effectués les mêmes jours et heures dans des conditions semblables de température, humidité et lumière, dans la même ferme, mais à $200 \mathrm{~m}$ de distance de la ruche, ainsi que séparément dans le centre de la ville.

Afin de compter les colonies microbiennes, on maintient les boîtes de Petri à l'étuve, à $37^{\circ}$ pendant 48 heures. Pour les moisissures, les boîtes sont gardées encore 4 jours à la température du laboratoire.

Ces expériences, effectuées en plein été 1963 , ont été répétées trois fois, approximativement à six jours d'intervalle. On a constaté le développement surtout $B$. sub- 
tilis mesentericus et Proteus vulgaris ; dans un nombre plus réduit de cas on a trouvé des colonies de staphylocoques et comme moisissures d'Aspergilus niger et de Mucor mucedis.

Le tableau 6 montre une moyenne de huit colonies de microbes et sept colonies de moisissures dans l'atmosphère interne du rucher, 2 I colonies de microbes et I 8 moisissures dans le verger situé à $200 \mathrm{~m}$ du rucher et 38 colonies de microbes et 7 de moisissures dans les prélèvements effectués en plein centre de la ville. Ces chiffres indiquent une réduction évidente de l'aéroflore dans l'ambiance du rucher comparativement à celle du verger situé à $200 \mathrm{~m}$; elle est encore plus accentuée par rapport à l'aéroflore de la ville qui marque toutefois un nombre plus réduit de colonies de moisissures.

\section{TABI,EAU 6}

L'aíroflore des divers centres: nucher, zerger, rille

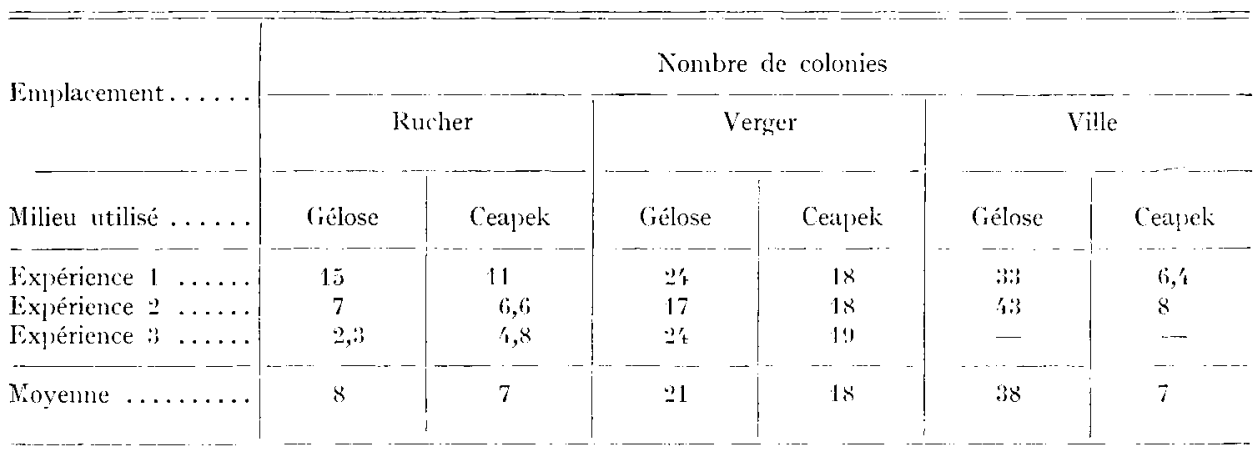

Les chiffres représentent le nombre de colonies obtenu par lecture après l'incubation de 6 boît es de l'étri exposées frendant 10 ninutes à l'atmosphère des différents lieux à différents moments de la joumée.

\section{CONCLUSIONS ET DISCUSSION}

Des expériences concernant l'action de la propolis, il résulte :

$\mathrm{I}^{\circ}$ L'extrait alcoolique de propolis soviétique, ajouté à une culture en bouillon, en proportion de $\mathrm{I} / \mathrm{IO}$, maintenu à $37^{\circ}$ après l'ensemencement, inhibe la culture de $S$. enteritidis GÄRTNER 5 I68, E. coli Bruxelles, E. coli $026 ; E$. coli orII, Sh. sonnei S., Sh. dysenteriae 2 HELLY, S. cholerae suis I79 phase I, S. typhiTOgor, Entérocoque (Str. faecalis). Il n'agit pas envers $S$. subtilis mesentericus, ni staphylocoque Oxford.

$2^{\circ}$ Après maintien du bouillon + propolis à $22^{\circ}$ (de Io minutes à 25 heures), on ne constate point d'action inhibitrice sur les microbes signalés antérieurement: $E$. coli oIII, S. cholerae suis. phase I, à l'exception de l'entérocoque (str. faecalis). Cette action se manifeste après 30 heures de contact de la culture de $E$. coli or I I er $S$. cholevae suis., avec la propolis soviétique et $\mathrm{R}_{2}$, intervalle pendant lequel la propolis $\mathrm{R}_{1}$ ne présente cette propriété que pour l'entérocoque (Str. faecalis). Nous soulignons que la propolis $R_{1}$ avait été soumise au malaxage et chauffée à $60^{\circ}$ pendant I5 minutes. 
$3^{\circ}$ L'action inhibitrice sur la germination des semences de chanvre (Canabis sativa) se manifeste à la concentration de $\mathrm{I} / \mathrm{Io}$ et $\mathrm{I} / 20$, en solution aqueuse des propolis "U. R. S. S. " et $R_{2}$, proportionnellement à la concentration, la propolis soviétique au $\mathrm{I} / \mathrm{I}$ o réduit de trois fois le taux de germination et la propolis roumaine I/Io de 6 fois.

$4^{\circ}$ L'aéroflore d'un rucher est comparativement beaucoup plus pauvre, avec une moyenne de 8 colonies, que celle d'un verger situé à $200 \mathrm{~m}$ de distance (2I colonies) ; la moyenne des colonies de moisissures est de 7 pour le rucher et r 8 pour le verger. L'aéroflore de la ville est beaucoup plus riche en bactéries qu'en moisissures. Celle du verger est la plus riche en moisissures.

Nos résultats démontrent les propriétés antibiotiques des échantillons de propolis roumaine $R_{1}$ et $R_{2}$, ainsi que de la propolis "U. R.S.S. ».

Rę̧u pour publication en juin 1964 .

\section{SUMMARY}

RESEARCH ON CHRTAIN BIOLOGICAL PROPJETIES OF PROPOLIS

Experiments have been undertaken which aimed at comparing the antibiotic properties of certain samples of propolis harvested in this country with those of the U.S.S.R.

An alcoholic extract was used, which was prepared by macerating the native propolis in 96 p. soo ethyl alcohol. The percentage of dry material obtained from the extracts of the various samples ranged from 6 to $7 \mathrm{~g}$.

The following were investigated :

1) The inhibitive effect of the alcoholic extract on cultures of certain microbes.

2) The effect on the germination of certain seeds.

3) The effect of the native propolis on the aeroflora of an apiary.

In the first series of experiments, the author found :

a) An inhibitive effect of the alcoholic extract of the U.S.S.R. propolis in a $\mathrm{I}:$ Io dilution on the following microbes : $E$. coli 1 RUXXLLES, S. enteritidis GARTNER $568, E$, coli 026 and 1111 , Sh. sonnei S., Sh. dysenteriae 2 HELIY, $S$. cholerae suis I 79 , phase I, S. typhi To gor, enterococcus (str. faecalis). There was no inhibitive effect on B. subtillis mesentericus, and the Oxford staphylococcus.

The results were obtained with tubes of broth + propolis sown with different microbes and kept at $37^{\circ} \mathrm{C}$, and subsequently transfered to agar-agar.

b) When tubes of broth $\frac{1}{+}$ propolis $\mathrm{R}_{1}$ were kept at $22^{\circ} \mathrm{C}$ before the transfer to agar-agar, the inhibitive effect on the Oxford staphylococcus, $F$. coli and or I 1, Sh. sonnei, Sh. dysenteriae 2 Ini.l. S. cholerae suis I 79, phase I, S. typhi To gor did not appear. 'This inhibition took place nevertheless starting from 4 hours, but only with Sh. dysenteriae 2 HELIY and enterococcus (Str. faecalis).

4) With a temperature of $37^{\circ} \mathrm{C}$, inhibition was shown in all series of microbes, with the exception of staphylococcus.

c) Comparative experiments on the three samples (U.S.S.R., $R_{2}$ and $R_{1}$ ) showed that the last product, which had been previously submitted to mixing and heating at $60^{\circ} \mathrm{C}$, had a weaker inhibiting effect.

The experiments concerning the inhibiting effect of propolis on the germination of certain seeds revealed an inhibiting effect on the seeds of hemp (Cannabis sativa), which occurred at a $1: 10$ dilution.

To study the influence of native propolis on the internal aeroflora of the apiary, comparative determinations were made on the aeroflora of an orchard situated $200 \mathrm{~m}$ from the hives, and on that of the centre of Bucharest. The investigation showed that there was a definite reduction of the acroflora of the apiary in relation to that of the orchard and of the town. There were few mould colonies both in the apiary and in the town.

It is concluded that the results demonstrate antibiotic properties in the propolis harvested in this country. 


\section{RÉFÉRENCES BIBLIOGRAPHIQUES}

Brtsilovski I. A., ig6o. La propolis comme moyen thérapeutique dans les maladies gynécologiques (en russe). Tesisi Docl. 2-a Leningradskaia nauk. Conf. Léningrad 1960, 55-56.

Cinsiser Z. G., ig60. Iat propolis, un moyen thérapeutique précieux (en russe). Ibil., 79.

Gracoleva E. V., ig6o. Sur l'utilisation de la propolis en médecine (en russe). Ibid., $77-78$.

Convet Il., Istvil: P., 1960. Action antigeminative des produits de la ruche d'abeilles (.tpis mellifica I..). C. R. Acad. Sci., 250, 61 $2-6 \mathbf{1 4}$.

Ijkisova C. G., igro. J'usage de la pommade avec propolis dans la nécrobacillose des animaux domestiques (en russe). Tesisi Dod. 2-a Leningradskaia natk. Cont. Leningrad, I960, 73.

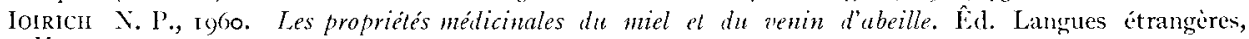
Moscou.

IoIrich N. P., Derevicl A., Petrescu A., 1964. Action virulicide in vitro d'un extrait de larves de faux-bourdon et d'un extrait de propolis (en roumain). Traz'aux sci. de la Station de Recherches apicoles et séricicoles Rommanie, Bucarest, 5, (sous pressic).

KARimova Z. II., s g6o. Ia propolis, un moyen thérapentique nouveau (en russe). Tesisi Docl. 2-a Leningralskaia nauk. Conj., leningrad, I960, 80-81.

Knzakov I. F., Aruzarov I. S. et Aristov A. A., ig6o. Ia thérapie des bovins malades de la fievre aphteuse avec une pommade préparée ave de la propolis (en russe). Ibit., 7o-7o.

Kazakor I. F., ARISTOY A. A., SAvisa K. A, ig6o. I a propolis, un moyen thérapeutique nouveau dans la médecine vétérinaire (en russe). Ibid., 68-69.

Kazakov I. F., Kuleev li. T., I960. Guérison des vaginites et vestibulites des vaches avec la pommade avec propolis (en russe). Ibill, 72

Keller li. İ., Prtdnicenko E. C., igho. Sur la composition de la propolis et l'activité bactéricide (en russe). Ibid., $53-54$.

Kinklior I. P., Kulikov N. S., ig6i. Traitement des Abeilles malades de la loque européenne avec de la propolis (en russe). Pcelovodstvo, 38, (г 2 ), $4 \mathbf{I}-+2$.

Kivalina V. P., I 960 . 'Thérapie à l'aide des médicaments à base de propolis et action antimicrobienne (en russe). Tesisi Docl. 2-a Leningradskata Nauk. Conf. Leningrad, 1960, 61-62.

Kun Eev F. T., Ig6o. L'usage de la pommade avec propolis en chirurgie vétérinaire (en russe). Ibill., $72-72$.

Lavie P., Ig6o. Les sulustances antibactériemes dans la colonie d'abeilles (Apis mellifica I..). Ann. Abeille, 3 (2), $103-197$.

Lavie I'., ig60. Les substances antibactériennes dans la colonic d'abeilles (Apis mellifica). Ann. Abeille. $3(3), 201-305$.

Marcexio A. I., Ig6o. L'usage de la propolis en stomatologie (en russe). Tesisi Dod. 2-a Leningradskaia Nauk. Conf., Leningrad, I960, $57-58$.

Marin M., Matelsco 'T., Bataci T., P'opa A., i959. Contribution à l'étude de la propolis (en roumain). Apicultura, 12, 9-16.

Huhamedin rof G. Z., ig6o. La propolis, un nouveau moyen de traitenent de l'eczéma (en russe). Tezisi Docl. 2-a Leningradekaia Nauk. Con/. Jeningrad, $1960,63^{-6.4}$.

RaBinovici I. M., igto. L'action bactéricide de la propolis sur le mycobacterium tuberculosis (en russe), Ibid., 59-6o.

Salmakov K. M., igto. L'action bactéricide de la propolis dans la I3rucellose (en russe). 1bid., 66-67.

SELARSki V. A., ig6o. Sur l'effet de la propolis sur les porcelets du kolkhoze Jurdinski (en russe). Ibid, $7+75$.

Selarski V. A., ig6o. Les résultats obtenus par l'usage de la propolis dans la fièvre aphteuse des vaches (en russe). Ibid., 76-76.

STEPanova M. A., I960. Les résultats thérapeutiques du venin d'abeille et de la propolis dans les maladies de la peau de l'enfant (en russe). Ibid., 65-65.

Verge J., 195I. Activité antibactérienne de la propolis, du miel tt de la gelée royale. Apiculleur, 95 (6), I3-2O. 